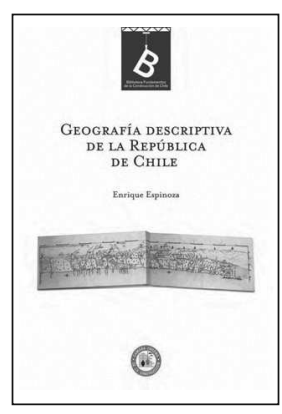

\section{Biblioteca fundamentos de la construcción de Chile. Obras de carácter geográfico que forman parte de la biblioteca.}

\author{
Santiago de Chile: Cámara Chilena de la Construcción, \\ Pontificia Universidad Católica de Chile y Dirección de \\ Bibliotecas, Archivos y Museos, 2008-2014.
}

José Ignacio González Leiva ${ }^{1}$
La Cámara Chilena de la Construcción de Chile (CChC), convocó el año 2007 a un concurso público denominado "Un Regalo para Chile", iniciativa con la que se pretendía adherir a la conmemoración del Bicentenario de la República de Chile. La Facultad de Historia, Geografía y Ciencia Política de la Pontificia Universidad Católica de Chile, en conjunto con la Biblioteca Nacional de la Dirección de Bibliotecas, Archivos y Museos (DIBAM) y, en alianza con el Comité de Infraestructura Pública de dicha Cámara, se adjudicaron el concurso con el proyecto Biblioteca Fundamentos de la Construcción de Chile, encargo que ha culminado exitosamente a mediados del 2014, con la publicación de los 100 volúmenes comprometidos para dar forma a la colección.

La Biblioteca, tuvo como propósito central la reedición de obras de científicos, profesionales y técnicos, publicadas desde los inicios de la formación de la república y hasta 1950. Publicaciones a través de las cuales dieron a conocer Chile, sus recursos humanos y naturales, así como las características sociales y evolución histórica, contribuyendo con sus investigaciones, informes y trabajos a la formación de la nación, la organización de

1 Instituto de Geografía, Pontificia Universidad Católica de Chile (Chile). E-mail: jigonzal@uc.cl la república, la administración del Estado y el desarrollo general del país.

Los objetivos específicos apuntaban en primer término a seleccionar los títulos que se ajustaran al propósito central del proyecto, para luego proceder a su edición y publicación, tarea que no resultó fácil de concretar dado los méritos de los más de 150 títulos preseleccionados y que a juicio del Comité Editor, merecían ser parte de la Biblioteca. En segundo lugar, se contemplaba que cada título fuese acompañado por un estudio introductorio realizado por un especialista en la materia, en el que se diera cuenta del autor de la obra, de la génesis del texto, su contenido, con especial énfasis en los temas que aborda, problemas y proposiciones que realiza, así como también se indicara la recepción del escrito en su época. De igual modo, se requería que el estudio introductorio pusiera en su contexto la trascendencia del trabajo, en aspectos tales como: la organización de la república, la formación de la nación, la explotación de los recursos naturales, el progreso material del país, el desarrollo social o cultural, el desenvolvimiento económico, el reconocimiento del territorio, etc., para finalmente, entregar una visión de su valor en el presente, como documento que permite conocer el pasado y proyectar el futuro. Un tercer aspecto a considerar, se relacionaba con producir una propuesta multimedia, mediante la concreción de un Sitio Web y videos, con 
el propósito de dar a conocer la Biblioteca y estimular su uso, en especial como material de apoyo para los establecimientos de enseñanza del país, fomentando así el aprovechamiento, conocimiento y lectura de las obras que componen la colección por parte de los estudiantes y público en general. En cuarto lugar, se consideraba la distribución de la colección en todas las bibliotecas públicas del país, liceos y universidades, para poner a disposición de sus usuarios estas obras clásicas fundamentales de difícil acceso, en las que se muestra la evolución científica, técnica y socioeconómica del país, donde queda de manifiesto la notable contribución de sus autores al patrimonio cultural de la nación.

Del total de obras que componen la $\mathrm{Bi}$ blioteca, merecen destacarse aquellas que se identifican con el quehacer de la ciencia geográfica, tanto por el número de publicaciones que pueden considerarse vinculadas a la disciplina, como por la importancia que ella alcanzó en la formación y organización de la nación chilena. En la geografía chilena del siglo XIX y hasta mediados del siglo XX, predominó un enfoque netamente descriptivo con algunos atisbos de análisis, cuyo objetivo primordial será ocuparse de los hechos y fenómenos que ocurren en la superficie terrestre, la que sirve de morada al hombre y de la cual este debe hacer un uso racional, con la finalidad de preservarlo para las generaciones futuras. Ciertamente, muchas de las obras incorporadas en la Biblioteca y que concebimos vinculadas con la geografía tienen esta connotación y gran parte de ellas no fueron realizadas por geógrafos propiamente tales, pero sí por profesionales de ciencias afines o bien técnicos interesados en asuntos espaciales relacionados con la población, los recursos, las actividades económicas, etc. Todos tienen en común, el mérito de haber contribuido de manera significativa al reconocimiento del territorio chileno, ya sea mediante encargos del gobierno central de la nación o bien por iniciativa propia, reconocimiento que en la mayoría de los casos es producto de viajes de exploración. Tales expediciones, se inician ya en los albores de la república durante su etapa de formación, cuando no se tenía conocimiento certero de la distribución y localización de los recursos naturales y humanos de los que disponía el país. Así como otros, que surgen en el momento de la consolidación y desarrollo de la nación, que aportaron vitales antecedentes para conocer la potencialidad de los recursos, como también para colaborar en el ordenamiento, organización y administración del espacio nacional. Junto a los trabajos anteriores, la Biblioteca recoge también otros estudios que tienen la virtud de sistematizar la información territorial, mediante manuales de geografía, estudios específicos de un área del país o anuarios estadísticos.

\section{Reconocimiento del territorio}

Entre las obras que surgen en los inicios de la República, relacionadas con el reconocimiento del territorio chileno, se encuentra una parte importante de los textos que conforman la monumental obra de Claudio Gay titulada "Historia Física y Política de Chile", realizada entre 1844 y 1871, compuesta por un total de treinta volúmenes, de los cuales 20 son obras relacionadas con el quehacer geográfico. En efecto, ocho de ellos corresponden a la "Botánica" (1845-1852), los que están destinados a presentar las características de la flora del país, donde se describen las familias, los géneros y las especies que reconoció junto a sus colaboradores en sus viajes a lo largo y ancho del territorio chileno. Hay también otros ocho volúmenes consignados a la "Zoología", en los que describe la fauna nacional, incluyendo tanto a los animales vertebrados como invertebrados. Para los vertebrados reunió información de las diferentes clases taxonómicas: mamíferos, aves, reptiles, anfibios y peces que son tratados en los tomos I y II de su Zoología, por su parte, los invertebrados son abordados en los tomos III, IV y V. Los insectos son descritos en los tomos VI y VII. El tomo VIII, está reservado a los moluscos; dos volúmenes los destina Claudio Gay para describir la actividad agrícola y su potencialidad futura, ya que este rubro de la economía lo consideraba el más importante del país y en el que además, pensaba que estaba fundado el futuro de Chile para llegar a ser una nación próspera. Otros dos tomos, están reservados a la cartografía del territorio, siendo ellos los primeros mapas levantados mediante la aplicación de métodos científicos, los que corresponden a un mapa general de Chile y a las respectivas provincias. Junto a ellos, hay una serie de láminas que muestran diversos paisajes chilenos y actividades 
de sus habitantes, cuyo conjunto conforman un Atlas. En consecuencia, de la obra de Gay, dos tercios de ella tienen una vinculación directa con la geografía chilena.

El interés y conocimiento por determinadas áreas del territorio nacional cobra especial relevancia durante esta época, motivadas por diversas razones, algunas de carácter económico, otras de tipo político así como también de orden social. Dentro de estas, destacan, entre otras, las relacionadas con la Araucanía, el desierto de Atacama y la Patagonia. En este contexto, se enmarcan las obras de Ignacio Domeyko "La Araucanía y sus habitantes" y la de Cornelio Saavedra "Documentos relativos a la ocupación de Arauco". La primera, publicada en 1845, rastrea las características de la naturaleza física y humana del territorio ocupado por el pueblo mapuche, emplazado entre el río Biobío por el norte y el Toltén por el sur, cuando aún la Araucanía mantenía su independencia. La obra de Cornelio Saavedra por su parte, se relaciona de manera directa con el proceso que se llevó adelante para incorporar este territorio a la estructura político administrativa vigente en el país. Es decir, crear en él una nueva provincia, lo que implicaba ocuparla militarmente y luego, Ilevar adelante la respectiva colonización, asunto que recoge este texto mediante una serie de informes y documentos, hasta su pacificación definitiva en 1883. Además de lo anterior, en tales documentos se hace referencia al modo de vida del pueblo mapuche, así como también a las características naturales del terreno, el cual fue reconocido primeramente con el nombre de Territorio Fronterizo de la Provincia de Arauco, el que luego del avance en su ocupación y reducción de su superficie pasó a denominarse Territorio de Colonización de Angol, enmarcado por la cordillera de $\mathrm{Na}$ huelbuta por el oeste y los ríos Renaico por el norte y el Cautín por el sur, espacio que finalmente dará origen a las provincias de Malleco y Cautín en 1887.

Por otra parte, también en el contexto de explorar territorios poco conocidos, se encuentra el desierto de Atacama, donde se inscribe el trabajo de Rodolfo Amando Philippi "Viaje al Desierto de Atacama", obra que corresponde a un encargo que el gobierno de Manuel Montt le hace a este naturalis- ta alemán el año 1853, cuya finalidad era conocer esta área inhóspita en sus diversas dimensiones, tanto naturales como humanas $y$, a la que se añadía otra de orden geopolítico por estar convirtiéndose en el centro de la controversia limítrofe que nuestro país mantenía con Bolivia. En este mismo orden de cosas, encontramos el trabajo de Francisco J. San Román, titulado "Desierto y Cordilleras de Atacama"; el texto reeditado por la Biblioteca, corresponde a una selección de los documentos elaborados por la comisión que se formó bajo el gobierno de Domingo Santa María, para explorar el desierto de Atacama, en el momento que la Guerra del Pacífico llegaba a su fin, y que se mantuvo en operación entre 1883 y 1890 . La finalidad de esta comisión de la que San Román era su Ingeniero Jefe, fue hacer un reconocimiento lo más exhaustivo posible del desierto, con especial atención en su orografía, hidrología, geología y recursos mineros, además del levantamiento de una carta topográfica detallada. Interesante resulta la visión que San Román trasmite de esta área, la que difiere un tanto de la dada a conocer por otros viajeros y exploradores, quienes la catalogaban como un espacio cuyas condiciones naturales dificultaban su habitabilidad, mientras que él valoró el desierto, como un espacio geográfico único y por su potencial minero, como fuente del desarrollo económico del país.

Referente al extremo sur del país, otra de las áreas desconocidas y que despertaban tanto un interés científico, como también por sus implicancias económicas, geopolíticas y de colonización, fue la Patagonia. En la Biblioteca se encuentran una serie de obras que aportaron valiosos antecedentes a su conocimiento y reconocimiento por parte de viajeros y exploradores, como la de Guillermo E. Cox, titulada "Viaje en las Regiones Septentrionales de la Patagonia 1862-1863". Ella trata de los viajes de exploración que realiza este médico en la Patagonia norte, donde describe las características de este espacio geográfico, aprovechando su conocimiento del área, producto de haberse desempeñado entre 1854 y 1856, como el primer administrador de la Colonia de Llanquihue, territorio cuyo conocimiento resultaba de especial interés para el gobierno del Presidente Manuel Montt, por las posibilidades de colonizarlo y por ser además, una zona de contacto con 
Argentina a través de los pasos cordilleranos existentes en torno del lago Nahuel Huapi. Del geógrafo alemán Hans Steffen, pertenece el trabajo en dos volúmenes titulado "Viaje de Exploración y Estudio en la Patagonia Occidental 1892-1902", el que responde a una solicitud de carácter oficial del gobierno chileno, con la finalidad de recoger antecedentes geográficos, con especial atención por los hidrográficos, y la realización de un levantamiento cartográfico del área, para enfrentar de mejor forma la disputa de la frontera austral que Chile mantenía con la República Argentina por la Patagonia. Sin embargo, los alcances de este trabajo fueron más allá, gracias al poder de observación del geógrafo germano, quien valora señeramente el potencial futuro de la región, al ponderar el área como un espacio adecuado para llevar adelante un proceso de colonización, así como también la riqueza de los recursos naturales disponibles, tanto los hidrológicos, ganaderos y agrícolas. Del padre salesiano Alberto M. de Agostini es también la obra, en dos tomos, publicada en 1945, destinada a este sector del país que se incluye en la Biblioteca, titulada "Andes Patagónicos. Viajes de Exploración a la Cordillera Patagónica Austral", en la que este incansable explorador vuelca todo su conocimiento acumulado, por más de cuatro décadas, en este trabajo reconocido como una notable contribución a la geografía de la parte austral de América, en la que da a conocer la estructura orográfica y glaciológica de los Andes Patagónicos y Fueguinos, lo que ilustra con abundantes fotografías y cartografía. A lo anterior se agregan las cuidadas referencias a sus habitantes, especialmente los alacalufes, a los que conoció y con los cuales compartió en el transcurso de su exploraciones, todo lo cual contribuye a hacer de esta obra un clásico dentro de la literatura geográfica, destinada al extremo austral de Chile y América.

\section{Manuales y compendios de geografía de Chile}

Entre los textos que podríamos catalogar como manuales o compendios dentro de los títulos que componen la Biblioteca, se encuentra la obra de Amado Pissis "Geografía Física de la República de Chile", publicada en 1875 y que es el resultado de la experien- cia del trabajo en terreno que él inicia a partir de 1848, momento en el que el gobierno chileno lo contrata y le encomienda por una parte, hacer un levantamiento de los recursos con los que cuenta el país, así como también la realización de un mapa topográfico de su territorio. En el texto, su autor reseña, en la parte correspondiente a lo que él denomina "Reino Inorgánico", las características físicas del país en cuanto a su orografía, geología, meteorología e hidrografía, con un marcado acento en los asuntos relacionados con la geología y los recursos mineros disponibles en el territorio chileno, lo que responde a su formación académica de geólogo. La segunda parte, la destina al "Reino Orgánico", que lo engloba bajo la denominación de "Geografía Botánica" y, en la que analiza brevemente la vegetación, agricultura y fauna de Chile. Forma parte también de la presente reedición, el atlas levantado por Pissis, formado por una serie de mapas topográficos correspondiente a cada una de las provincias de Chile, acompañada de un conjunto de gráficos y paisajes del territorio nacional.

La obra de Enrique Espinoza G., titulada "Geografía Descriptiva de la República de Chile", es otro de los títulos que caen dentro de esta categoría. Fue publicada por primera vez en 1890 y contó con cinco reediciones. La reeditada en la colección de la Biblioteca corresponde a la cuarta edición del año 1897. Se trata de un compendio de la geografía de Chile de fines del siglo XIX, la que como su nombre lo indica, es una descripción sistemática, ordenada y detallada de las características del territorio chileno, en sus aspectos físicos, económicos, culturales y sociales, con abundante información estadística y acompañada de una cuidada cartografía, en la que destaca la destinada a mostrar el ámbito territorial de cada una de las 23 provincias y un territorio (el de Magallanes), en la que se encontraba dividido el país desde el punto de vista político-administrativo, en el momento de su publicación. De ella se sirve para reseñar cada provincia en cuanto a su superficie, población, recursos naturales, centros urbanos, vías de comunicación, entre otros aspectos. Incluye, como novedad, en una obra destinada a la geografía de Chile de esta época, las provincias de Tacna, Tarapacá y Antofagasta, recientemente incorporadas al 
territorio chileno, luego de finalizada la Guerra del Pacífico.

La "Geografía Botánica de Chile" es obra del botánico alemán Karl Reiche, escrita originalmente en el idioma de su autor el año 1907, en ella reseña la flora y vegetación de Chile, luego de su permanencia en el país por cerca de 17 años. Este escrito titulado "Grundzüge der Pflanzenverbreitung in Chile" (Principio de la distribución de las plantas en Chile) pasó a ser parte de la famosa serie: "Die Vegetation der Erde" (La Vegetación del Mundo) de Adolf Engler y Oscar Drude, la cual es traducida posteriormente en 1934 por Gualterio Looser, bajo el título de "Geografía Botánica de Chile", siendo esta traducción la que se reedita en la Biblioteca. La obra es una fuente de la mayor importancia, no solo para los botánicos y profesionales de ciencias afines, sino que también para estudiantes y público en general, los que a través de su lectura podrán reconstituir los ecosistemas que existían en el territorio chileno a principios del siglo XX, los que con especial maestría describe Reiche en sus páginas, paisajes botánicos que lamentablemente a través de los años fueron desapareciendo. El mérito de la obra en referencia, radica en el hecho que si bien existían en Chile descripciones de la vegetación, como las de Claudio Gay y Rudolfo Philippi, Reiche integra los aspectos vegetacionales con los florísticos, lo que viene a llenar un vacío existente en este aspecto. Cabe añadir, el valor histórico y geobotánico de la cartografía que acompaña su escrito, así como también el aporte de los autores del estudio introductorio, quienes actualizaron los nombres científicos utilizados en la obra.

La "Geografía Náutica de Chile" de Francisco Vidal Gormaz, forma parte de la colección de la Biblioteca y en ella se reúne lo que su autor publicó entre 1879 y 1884 en el "Anuario Hidrográfico de la Marina de Chile". A lo largo de sus páginas, describe la costa chilena desde la frontera norte hasta Chiloé, en ella analiza las características propias de la hidrografía maritima y fluvial, en relación con las necesidades propias de la navegación. La obra está acompañada de importante material cartográfico levantada por el propio Vidal Gormaz. No obstante, ser una obra destinada a la navegación, su autor incursiona también en asuntos vinculados con la población, los recursos naturales, las actividades económicas, la historia de los lugares visitados en sus viajes de exploración a lo largo de la costa chilena y su incursión por los ríos navegables, material que complementa con antecedentes disponibles en la Oficina Hidrográfica, de la que fue su Director a partir de 1874.

La "Geografía Económica de Chile", de la Corporación de Fomento de la Producción, CORFO, es el compendio más completo de geografía de mediados del siglo XX, realizada con el propósito de entregar una visión integral del territorio chileno que permitiera sentar las bases para impulsar el desarrollo económico y social de la nación, situación en la que se reconoce que la geografía juega un papel trascendental. Dicho objetivo se logró plenamente, lo que le valió a este trabajo haberse constituido en una obra clásica de la geografía chilena, por su enfoque analítico e integrado bajo la cual se estructuró. Es una obra compuesta por cuatro tomos: el primero está destinado a mostrar los factores naturales del país, el segundo a los factores humanos, el tercero se dedica al estudio de las diversas ramas de la producción y el cuarto, corresponde a una monografía de cada una de las seis regiones, las que estaban basadas en el criterio de su homogeneidad territorial desde el punto de vista económico-geográfico, a saber: Norte Grande, Norte Chico, Núcleo Central, Concepción y La Frontera, Región de Los Lagos y Región de Los Canales.

En este mismo grupo, se encuentra "El Anuario de la República de Chile". Estudios sobre Territorio y Población. La publicación que forma parte de la Biblioteca, pertenece a una selección de documentos publicados por la Oficina de Estadística entre los años 1860 y 1872 . Esta entidad creada en 1843, tuvo como finalidad recoger información confiable y fidedigna por parte del Estado, para generar estudios que fuesen un soporte para emprender de lo que hoy se reconoce como la elaboración de políticas públicas. Poco a poco fue avanzando esta publicación en calidad y prestigio, pasando de ser un instrumento descriptivo a uno más analítico. Esta etapa de transición y consolidación de la institución, es la que recogen los documentos aquí seleccionados. La vinculación del Anuario Estadístico con la geografía, viene dado por el 
hecho que incluye en sus páginas los asuntos relativos a las características del territorio, su población y los recursos con los que cuenta la república y como siempre utilizando como unidad territorial la provincia, todos elementos esenciales para el quehacer geográfico.

"Chile Ilustrado", es la obra que publica en 1872 Recaredo Santos Tornero, con la finalidad de mostrar a los chilenos y al mundo los logros alcanzados por nuestro país, en un momento de fuerte expansión de su desarrollo, lo que se reflejaba en los avances que eran posible reconocer mediante su infraestructura urbana y en el modo de vida que habían alcanzado de sus habitantes, todo lo cual constituía para el autor del trabajo motivo de orgullo nacional. Este recuento, lo efectúa con un enfoque espacial, al caracterizar cada una de las quince provincias y un territorio, el de Magallanes, en que se dividía el país, en el momento de su publicación, desde el punto de vista político-administrativo, siendo la provincia de Santiago la tratada con mayor detalle. La obra está acompañada una gran cantidad de ilustraciones correspondiente a edificios públicos y actividades de la población.

\section{Monografías geográficas}

Finalmente, cabe añadir a los títulos reseñados anteriormente, algunas monografías de carácter geográficas incluidas en la Biblioteca, como los dos títulos pertenecientes al naturalista alemán Ludwig Darapsky G.: "Las aguas minerales de Chile" y "El Departamento de Taltal". La primera, publicada el año 1890, corresponde a un estudio de la composición química de las fuentes termales que dispone el país y de sus bondades para la cura de diversas enfermedades, de las que deriva indicaciones y sugerencias para el diseño de políticas públicas relativas a la salud de la población. En su análisis Darapsky, incorpora la distribución geográfica donde las fuentes termales se localizan, diferenciando las pertenecientes a las de plena cordillera, ubicadas por sobre los 1.300 metros sobre el nivel del mar y las de entrada de la cordillera, emplazadas bajo la cota anterior y los 300 metros, es decir, la depresión intermedia y las de la región de la costa. Caracteriza cada una de ellas haciendo referencia a su situación, clima imperante, su acceso, etc. Este trabajo se inscribe, al igual que muchas otras obras de naturalistas, científicos y exploradores del siglo XIX, dentro de las de inventario de las riquezas naturales con que cuenta el país. El segundo trabajo de Darapsky, es una monografía referente al departamento de Taltal, provincia de Antofagasta, destinada a dar cuenta tanto de su historia natural como humana, la que realiza luego de su permanencia por cerca de una década en la ciudad de Taltal, y que fuera publicada en la ciudad de Berlín, en el año 1900. En ella, hace una notable descripción del relieve que impera en el desierto y destaca el grandioso esfuerzo realizado por los exploradores y mineros para enfrentar este paisaje agreste, carente de vegetación y agua, pero a su vez, pone de relieve la riqueza del desierto por sus yacimientos salitreros y los recursos mineros de que dispone. Por otra parte, y como consecuencia de su amplio conocimiento del área, producto de haber recorrido ampliamente el desierto y la puna de Atacama, elaboró un excelente mapa del desierto de Atacama y otro del departamento de Taltal, gracias a sus registros $y$ mediciones.

Al naturalista alemán Federico Albert, se deben las obras "Las dunas del Centro de Chile" y "Plan general para el cultivo de los bosques", que forman parte también de la Biblioteca. La destinada a tratar las dunas, Albert centra su atención en el litoral de las localidades de Constitución y Llico, sector al que es enviado por el gobierno para realizar un diagnóstico, destinado a determinar la relación existente entre el deterioro de los campos de cultivo y los bosques del sector y la invasión de arenas provenientes de las dunas costeras. A partir de su exhaustivo análisis, propuso como solución al problema presentado, un plan de manejo basado en la forestación para la contención del avance de las dunas, basado en la experiencia internacional sobre esta materia, asunto que recoge la publicación en referencia. Además este trabajo tiene un enorme valor para el quehacer de geografía teórica y aplicada, ya que por una parte comporta un detallado análisis de las dunas en cuanto a su origen, formación, extensión, modelos de defensa, tanto naturales como artificiales, etc., y, por otro, la perspectiva aplicada que tiene la ciencia geográfica en un problema territorial. Ambas vertientes, 
la teórica y la aplicada, que aborda este texto, lo han llevado a ser considerado una obra de referencia obligada para los estudiosos de la zona costera, tanto por su legado como por su valor científico del más alto nivel. La otra obra de Albert, que se reedita en la Biblioteca, reúne una serie de trabajos de su autoría, en los que muestra aquel rasgo que los distingue de manera excepcional, por la preocupación de los más variados aspectos ambientales, como la forestación, las dunas, los salmónidos y ciertamente el manejo de los bosques, entre otros, lo que sin duda proviene de su formación académica como doctor en Ciencias Naturales. Los estudios aquí reunidos, se han concentrado bajo el título de "Plan general para el cultivo de bosques", trabajo que publica el año 1907, mientras se desempeñaba como jefe de la sección de aguas y bosques del Ministerio de Industria y Obras Públicas, al que se han añadido, entre otros, La necesidad urgente de crear una inspección general de bosques, pesca y caza; los siete árboles forestales más recomendados para el país, los bosques de Chile, el problema forestal de Chile. Todos ellos contribuyen aún más a consolidar su destacada posición, en el manejo y uso eficiente de los recursos naturales del país, por lo que puede ser considerado con justicia "el padre de los estudios ambientales de Chile". 